\title{
Selective deletion of PPAR $\beta / \delta$ in fibroblasts causes dermal fibrosis by attenuated LRG1 expression
}

\author{
Ming Keat Sng ${ }^{1,2}$, Jeremy Soon Kiat Chan ${ }^{1}$, Ziqiang Teo ${ }^{1}$, Terri Phua ${ }^{1,3}$, Eddie Han Pin Tan', Jonathan Wei Kiat Wee', \\ Nikki Jun Ning Koh', Chek Kun Tan², Jia Peng Chen², Mintu Pal', Benny Meng Kiat Tong ${ }^{5}$, Ya Lin Tnay ${ }^{5}$, Xuan Rui $\mathrm{Ng}^{2}$, \\ Pengcheng Zhu', Shunsuke Chiba ${ }^{5}$, Xiaomeng Wang ${ }^{2,6,7,8}$, Walter Wahli2, ${ }^{2,10}$ and Nguan Soon Tan (1),2,6,11
}

\begin{abstract}
Connective tissue diseases of the skin are characterized by excessive collagen deposition in the skin and internal organs. Fibroblasts play a pivotal role in the clinical presentation of these conditions. Nuclear receptor peroxisomeproliferator activated receptors (PPARs) are therapeutic targets for dermal fibrosis, but the contribution of the different PPAR subtypes are poorly understood. Particularly, the role of fibroblast PPAR $\beta / \delta$ in dermal fibrosis has not been elucidated. Thus, we generated a mouse strain with selective deletion of PPAR $\beta / \delta$ in the fibroblast (FSPCre-Pparb/d ${ }^{-/-}$) and interrogated its epidermal and dermal transcriptome profiles. We uncovered a downregulated gene, leucine-rich alpha-2-glycoprotein-1 ( $\operatorname{rg} 1)$, of previously unknown function in skin development and architecture. Our findings suggest that the regulation of $\operatorname{Lrg} 1$ by PPAR $/ \delta$ in fibroblasts is an important signaling conduit integrating PPAR $\beta / \delta$ and TGF $\beta 1$-signaling networks in skin health and disease. Thus, the FSPCre-Pparb/d ${ }^{-1-}$ mouse model could serve as a novel tool in the current gunnery of animal models to better understand dermal fibrosis.
\end{abstract}

\section{Introduction}

Connective skin diseases are commonly characterized by excessive deposition of collagen in the skin and internal organs ${ }^{1,2}$. The classical hallmark of collagen overproduction is caused by myofibroblasts that are undergoing a dysregulated balance of signaling mediators, including cytokines and growth factors, resulting in dermal fibrosis that is observed in such diseases ${ }^{3}$. A prototypical and well-studied example of a connective skin disease is scleroderma (SSc). To date, treatments for these conditions exist only to provide symptomatic reliefs and helps to prevent further complications ${ }^{4}$. The precise etiology for the various clinical manifestations, however,

\footnotetext{
Correspondence: Ming Keat Sng (mksng@ntu.edu.sg) or Nguan Soon Tan (nstan@ntu.edu.sg)

${ }^{1}$ School of Biological Sciences, Nanyang Technological University, 60 Nanyang Drive, Singapore 637551, Singapore

${ }^{2}$ Lee Kong Chian School of Medicine, Nanyang Technological University, Novena Campus, 11 Mandalay Road, Singapore 308232, Singapore
} Full list of author information is available at the end of the article remains unclear. Many animal models have been developed to better understand the disease pathogenesis ${ }^{5,6}$. Although each animal model could not completely recapitulate all aspects of connective tissue diseases, these models collectively have advanced our understanding of the different aspects of these conditions. Importantly, they have underscored that connective tissue diseases, and the underlying dermal fibrosis and overproduction of collagen, are multigenic and involves communications among key cell types, such as keratinocytes, fibroblasts, and immune cells ${ }^{5-7}$.

Nuclear receptors (NRs) represent one of the largest classes of transcription factors that are also known to be druggable targets from the clinical perspective ${ }^{8-10}$. A variety of skin diseases is currently treated with FDAapproved drugs that target NRs, such as glucocorticoids (cortisone) and vitamin D (calcitriol) ${ }^{11}$. There is a total of 48 NRs in humans, yet only a handful of them have welldefined roles in dermal fibrosis and collagen 
overproduction, and thus are unexplored therapeutic targets for these conditions ${ }^{12}$. The discovery of peroxisome-proliferator activated receptors (PPARs) has attracted attention due to their crucial role in lipid homeostasis and tissue repair, ${ }^{8,11}$. There are three PPAR subtypes: PPAR $\alpha, \beta / \delta$, and $\gamma$. A recent study showed that

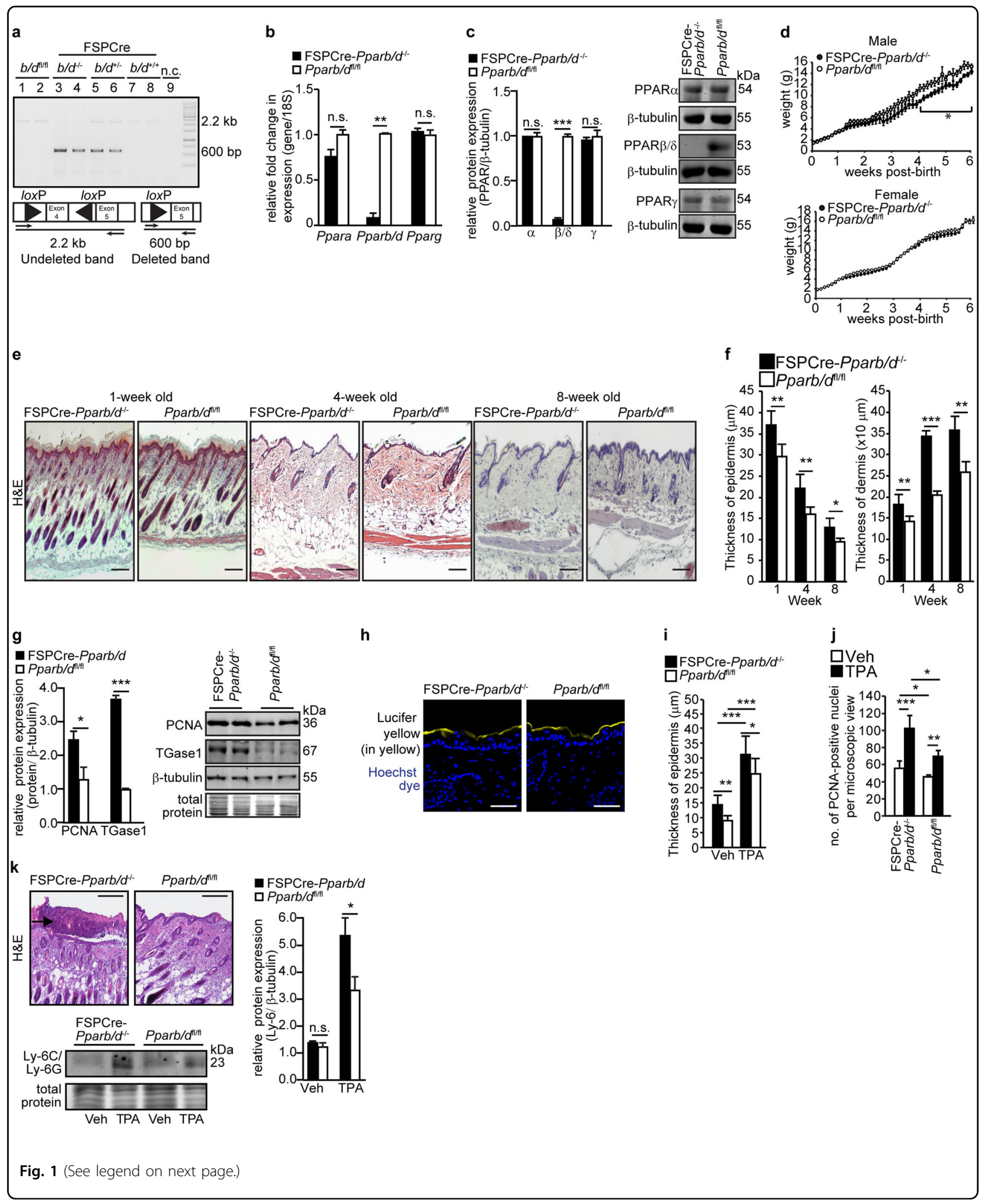


(see figure on previous page)

Fig. 1 Pparb/d ablation in fibroblasts enhances epidermal inflammation. a PCR analysis of mouse fibroblast DNA. The non-deleted floxed allele is represented by the $2.2-\mathrm{kb}$ band. The Pparb/d-deleted allele is represented by the 600 -bp band. n.c., negative control. b, c Relative PPARa, PPARß/ $\delta$, and PPARY mRNA (b) and protein (c) levels in fibroblasts of FSPCre-Pparb/ $d^{f / / f}$ and Pparb/d//fl mice. Representative immunoblots are shown. 18S rRNA served as housekeeping gene. $\beta$-tubulin that served as housekeeping protein was from the same samples. Values are mean \pm S.D. $(n=5)$. $\mathbf{d}$ Body weight of male and female mutant mice over 6 weeks post birth. Values represent mean \pm S.D. ( $n=35$ for male and $n=40$ for female mice). e Representative hematoxylin and eosin (H\&E) stained skin sections from FSPCre-Pparb/ $d^{A / f}$ and Pparb/ $d^{A / f l}$ mice at weeks 1,4 , and 8 of age. Scale bar $=$ $50 \mu \mathrm{m}$. $(n=12)$. $\mathbf{f}$ Epidermis and dermis thickness in both genotypes at weeks 1,4 , and 8 . Values represent mean \pm S.D. $(n=31)$. $\mathbf{g}$ Relative protein expression of epidermal markers for proliferation (PCNA) and differentiation (TGase1) in FSPCre-Pparb/d//f/7 and Pparb/d//7/ mice at age week 4. Representative immunoblots were shown. Coomassie stained of blots showed equal loading and transfer. $\beta$-tubulin that served as housekeeping protein was from the same samples. Values represent mean \pm S.D. $(n=5)$. $\mathbf{h}$ Barrier function test. Representative images of skins from FSPCre-Pparb/ $d^{f / f}$ and Pparb/d/f/A mice topically treated with Lucifer yellow dye. Nuclei were stained with Hoechst dye (blue). Scale bar $=50 \mu \mathrm{m}$. i, $\mathbf{j}$ Graphs showing the epidermal thickness (i) and the number of PCNA-positive keratinocytes (j) on dorsal skin treated with either vehicle (Veh) or $6.5 \mathrm{nmol}$ of 12-0tetradecanoylphorbol-13-acetate (TPA) for $24 \mathrm{~h}$. Values represent mean \pm S.D. $(n=8)$. $\mathbf{k}$ Representative H\&E stained section of TPA-treated skins from FSPCre-Pparb/ $d^{f / f l}$ and Pparb/d/f/f mice. Intra-epidermal microabscesses were observed in skins from FSPCre-Pparb/d//fl mice. Scale bar $=200 \mu \mathrm{m}$. Graph showing the relative protein expression of neutrophil marker Ly-6C/Ly-6G in Veh-treated and TPA-treated skin of FSPCre-Pparb/ $d^{A / f l}$ compared to Pparb/ $d^{f / f}$ mice. Representative immunoblots are shown. Coomassie stained of blots show equal loading and transfer. Values represent mean \pm S.D. $(n=5) .{ }^{*} P<0.05 ;{ }^{*} P<0.01 ;{ }^{* *} P<0.001 ;$ n.s. not significant

pan PPAR agonist IVA337 was effective in the prevention and treatment of experimental skin fibrosis ${ }^{13}$. The study pointed to a role for PPAR in dermal fibrosis, however, the contribution of various PPAR subtypes remains unclear. The skin of whole-body PPAR $\gamma$ heterozygous and fibroblast-specific PPAR $\gamma$-deficient mice did not exhibit any significant alterations in skin thickness or matrix accumulation. Nonetheless, the deletion of PPAR $\gamma$ resulted in enhanced susceptibility to bleomycin-induced skin fibrosis and specific agonists of PPAR $\gamma$ have been shown to alleviate the extent of the development of cutaneous sclerosis $^{14,15}$. As the most dominant PPAR subtype in the skin, the role of epidermal PPAR $\beta / \delta$ in skin physiology is well-documented $^{16-19}$. Sclerodermic keratinocytes can trigger an inflammatory response from the fibroblasts via secreted IL- $1 \alpha^{20,21}$. Interestingly, fibroblast PPAR $\beta / \delta$ has been shown to curb excessive epidermal proliferation during wound healing via the production of secreted IL-1 receptor antagonist ${ }^{22}$. Several studies have suggested that cellular redox state may play a significant role in the progression of scleroderma fibrosis ${ }^{23,24}$. Fibroblasts obtained from the skin of patients with SSc showed increase in reactive oxygen species production when compared to normal fibroblasts ${ }^{25}$. Incidentally, Wang et. al. underscored a novel role of fibroblast PPAR $\beta / \delta$ in the modulation of oxidative stress in a diabetic wound microenvironment ${ }^{26}$. These findings suggested a possible role for fibroblast PPAR $\beta / \delta$ in dermal fibrosis. However, whole-body PPAR $\beta / \delta$ knockout mice do not exhibit any dermal fibrotic phenotype ${ }^{8,27-29}$, and this may be because of a tissue-specific role for fibroblast PPAR $\beta / \delta$.

To understand the role of PPAR $\beta / \delta$ in fibrotic skin diseases, we generated a fibroblast-selective PPAR $\beta / \delta$ deleted mouse (FSPCre-Pparb/d $d^{-1-}$ ). FSPCre-Pparb/d $d^{-1-}$ mice exhibited profound morphological changes to the skin architecture at an early age, pronounced wound contraction and have altered gene expression profile that resembles fibroproliferative diseases.

\section{Results \\ PPAR $\beta / \delta$-deficient fibroblasts enhance epidermal hyperplasia and inflammation}

We generated a mouse whose exons 4-5 of PPAR $\beta / \delta$ gene were deleted in the fibroblasts by crossing Pparb/d $/ d^{\mathrm{f} / \mathrm{fl}}$ with FSP1-Cre mice, herein called FSPCre-Pparb/d ${ }^{-1-}$. The excision of $P$ parb $/ d^{\mathrm{f} / \mathrm{fl}}$ was identified by the amplification of a 600-bp DNA fragment in dermal fibroblasts isolated from FSPCre-Pparb/d -1- $^{-}$and FSPCre-Pparb/ $d^{+/-}$mice (Fig. 1a). Intact floxed Pparb/d ${ }^{\mathrm{f} / \mathrm{fl}}$ appeared as a 2.2-kb band (Fig. 1a). Multiplex PCR of CD $45^{+}, \mathrm{CD} 31^{+}$, and $\mathrm{MHC}^{+}$revealed differential deletion of PPAR $\beta / \delta$ allele in these isolated cells. No deleted PPAR $\beta / \delta$ allele was observed in either $\mathrm{CD} 31^{+}$or $\mathrm{MHC}^{+}$cells, whereas PPAR $\beta / \delta$ was deleted in $C D 45^{+}$cells (Fig. S1a). PPAR $\beta / \delta$ mRNA and protein were not detected in fibroblasts from FSPCre-Pparb/d $d^{-1-}$ mice (Fig. 1b, c). The relative expression of PPAR $\alpha$ and PPAR $\gamma$ in FSPCre-Pparb/d $/ d^{-1-}$ fibroblasts was not altered when compared to that in Pparb/d $d^{\mathrm{fl} / \mathrm{fl}}$ fibroblast (Fig. 1b, c). This was similarly observed in human dermal fibroblasts whose endogenous PPAR $\beta / \delta$ was suppressed by siRNA (Fig. S1b). The deletion did not affect the number of pups per litter or gender ratio (Fig. S1c). The body weight of male offspring was significantly lower than that of their wildtype counterparts, but this difference was not observed in female offspring (Fig. 1d, S1d). Thus, the FSPCre-Pparb/d ${ }^{-1-}$ mouse is viable and PPAR $\beta / \delta$ expression in fibroblasts is not critical for embryonic development.

To assess whether the FSPCre-Pparb/d $d^{-1-}$ mouse exhibited post-natal alterations, we performed histomorphometric analysis of the skin. The FSPCre-Pparb/d $d^{-1-}$ mice exhibited a thicker epidermis, dermis, and 


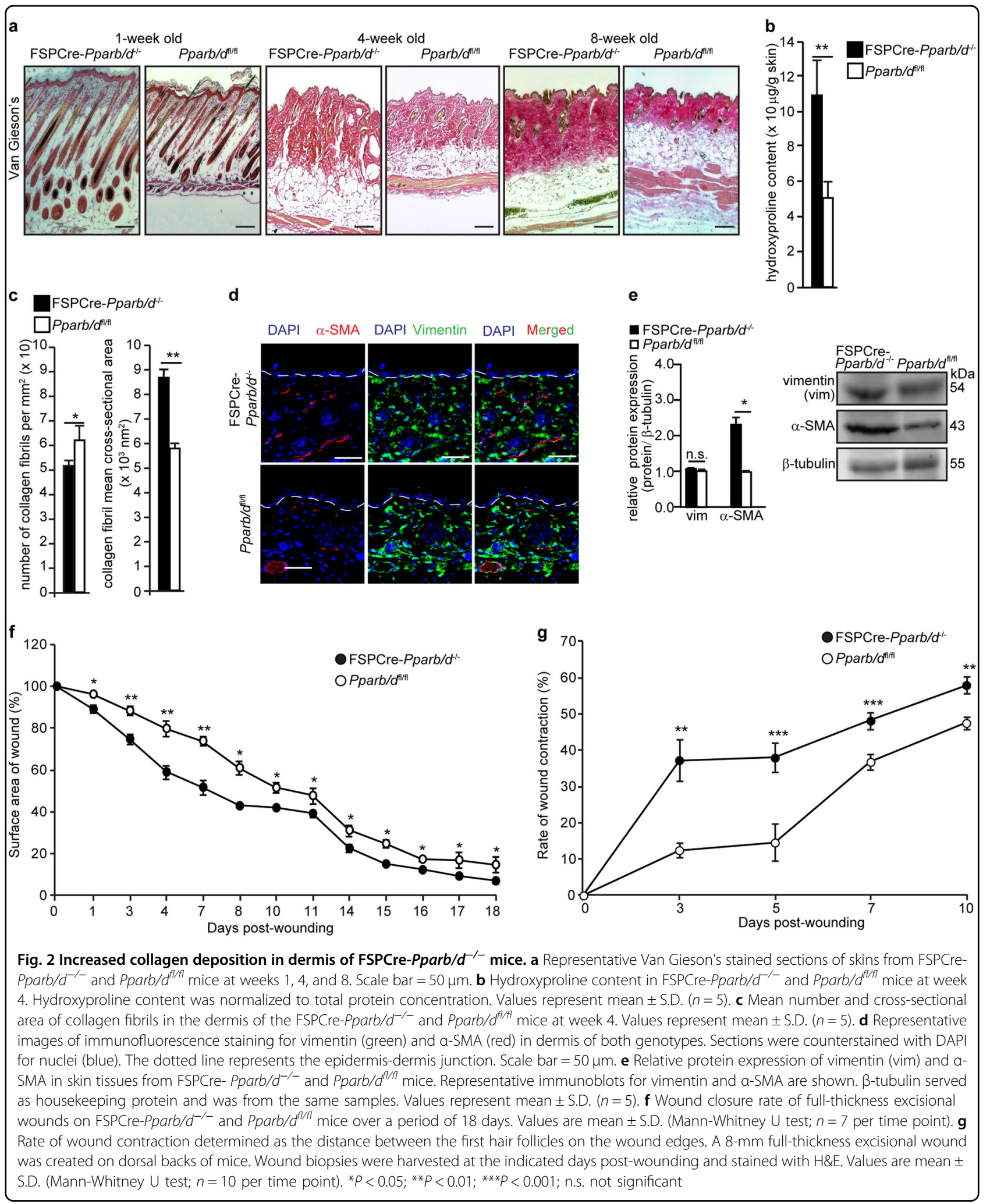

hypodermis (Fig. 1e, f, S1e). Immunohistochemical staining and western blot analysis revealed an elevated expression of PCNA (proliferation marker) as well as transglutaminase type 1 (TGase1; differentiation marker), suggesting an increase in the number of keratinocytes that proceeded to differentiate (Fig. 1g, S1f-g). We did not 
detect differences in the penetration of Lucifer Yellow dye into the epidermis of FSPCre-Pparb/d $d^{-/-}$and Pparb/d $\mathrm{d}^{\mathrm{I} / \mathrm{fl}}$ mice (Fig. 1h), suggesting that the skin barrier function was unaffected. Although, FSP1 has been reported to be a key marker of a specific subset of macrophages in the liver during fibrosis and injury ${ }^{30}$, the FSPCre-Pparb/d ${ }^{-1-}$ mice neither exhibited hepatomegaly nor had increased collagen deposition in the liver or gastrointestinal tract (Fig. S1h-i).

Previous studies showed that global PPAR $\beta / \delta$ knockout mice had an enhanced epidermal hyperplasia response when topically treated with 12-O-Tetradecanoylphorbol13-acetate (TPA) compared to their cognate wild-type littermates ${ }^{27,28}$. We questioned the role of fibroblast PPAR $\beta$ / $\delta$ in this acute TPA response, thus FSPCre-Pparb/d $d^{-1}$ and Pparb/d $d^{\mathrm{fl} / \mathrm{fl}}$ mice were treated with TPA. We observed an enhanced epidermal hyperplasia with more PCNA-positive proliferating keratinocytes in FSPCre-Pparb/ $/ d^{-1-}$ mice compared to Pparb/d $d^{f l f l}$ mice in response to topical TPA treatment (Fig. 1i, j, S1j-k). FSPCre-Pparb/d ${ }^{-/}$mice also exhibited intra-epidermal microabscesses with greater infiltration of Ly-6C/Ly-6G-positive neutrophils (Fig. 1k, S1l). Thus, PPAR $\beta / \delta$ ablation in fibroblasts led to an aggravated inflammatory response of the adjacent epidermis to TPA.

\section{FSPCre-Pparb/d ${ }^{-1-}$ mice exhibit dermal thickening and faster dermal wound healing}

Dermal thickening was the most obvious phenotypic difference between FSPCre-Pparb/d $d^{-/-}$and Pparb/d $d^{\mathrm{t} / \mathrm{fl}}$ mice. There was more collagen deposition in the FSPCrePparb/d $d^{-/-}$dermis compared with Pparb/d $d^{\mathrm{f} / \mathrm{fl}}$ as indicated by Van Gieson's stain and hydroxyproline measurement (Fig. 2a, b). We also stained the tissues using PicroSirus Red, which is intended for use in the histological visualization of Type I and III collagen in red and the cytoplasm in yellow. When viewed under polarized light microscope, a red fluorescence indicates the presence of Type 1 collagen (Fig. S2a). Consistently, we observed a thicker dermis and more collagen in the FSPCre-Pparb/d ${ }^{-/-}$ compared with Pparb/d $d^{\mathrm{f} / \mathrm{fl}}$ mice (Fig. S2a). Focused ion beam scanning electron microscopy of the dermis region revealed a larger mean cross-sectional area but fewer collagen fibrils in FSPCre-Pparb/d $d^{-1-}$ mice compared to Pparb/d $d^{\mathrm{f} / \mathrm{fl}}$ (Fig. 2c, S2b). We also detected more $\alpha$ smooth muscle actin ( $\alpha$-SMA)-positive myofibroblasts, but not vimentin-positive fibroblasts in FSPCre-Pparb/d $d^{-1-}$ mice compared to Pparb/d $d^{\mathrm{f} / \mathrm{fl}}$ mice (Fig. 2d, e). These findings suggest that $P P A R \beta / \delta$ deficiency in fibroblasts promotes the activation of fibroblasts to myofibroblasts, leading to increased dermal collagen deposition. To study how this phenotype affects dermal wound healing, we performed full-thickness excisional wounding assays. FSPCre-Pparb/d ${ }^{-1-}$ mice exhibited more rapid wound closure than the Pparb/d $/ d^{\mathrm{f} / \mathrm{fl}}$ mice (Fig. 2f, S2c), which was associated with a significantly higher rate of wound contraction (Fig. 2g, S2d). These observations suggest that the higher number of myofibroblasts and increased collagen deposition with thicker collagen fibers contributed to the rapid wound contraction in FSPCre-Pparb/d ${ }^{-1-}$ mice.

\section{FSPCre-Pparb/d ${ }^{-1-}$ mice exhibit a pro-fibrotic gene expression profile}

The above observations suggest that FSPCre-Pparb/d d- $^{-1}$ mice exhibited certain localized SSc-like hallmarks, including excessive collagen deposition leading to thickening of the dermis and greater number of myofibroblasts. Previous studies also concluded that localized SSc involves autoimmune abnormalities with elevated levels of anti-nucleosome antibodies ${ }^{31,32}$. We detected higher levels of anti-nucleosome antibodies in 69\% of our FSPCre-Pparb/d $d^{-1-}$ mice, with a higher frequency in female mice than male mice (Fig. S2e). To further strengthen our findings, we performed comparative microarray gene expression analysis of skin biopsies from SSc patients, other mouse models of SSc and FSPCrePparb/d ${ }^{-1-}$ mice.

An intrinsic SSc gene set comprising 995 genes was previously identified by Milano et al (GSE9285) ${ }^{33}$. We examined the expression of these genes in the tight skin (Tsk) 1 or 2 heterozygous mutant mouse model (GSE71999), the bleomycin-induced fibrosis mouse model (GSE71999), the sclerodermatous graft versus host disease (SGVHD) mouse model (GSE24410), and our FSPCrePparb $/ d^{-1-}$ mice (Fig. S3). Unguided hierarchical clustering of samples revealed that the mouse models of SSc mainly represented the fibroproliferative subtypes of human SSc. Both fibroproliferative 1 and 2 subtypes of human SSc share a common biological program of proliferation $^{33-35}$. FSPCre-Pparb/d $d^{-1-}$ mice samples branched off from the same node as fibroproliferative 2 subtype of human SSc samples, indicating that the gene expression profiles of the two sample groups are highly similar (Fig. S3).

A subset of 113 genes involved in cell:cell signaling and interaction, connective tissue development and function, and inflammation was downregulated in fibroproliferative human SSc compared with normal human skin (Fig. 3a). On the one hand, only our FSPCre-Pparb/d $d^{-1-}$ mice recapitulated this molecular signature of the diseased tissue, whereas these 113 genes were mostly upregulated in the Tsk1/2, SGVHD and bleomycin-induced mouse models of SSc. On the other hand, a subset of 110 genes that was upregulated in human SSc samples was better represented by Tsk1/2, SGVHD and bleomycin-induced mouse models of SSc than FSPCre-Pparb/d $d^{-1-}$ mice (Fig. 3b). However, we noted that the gene ontologies (GO) of deregulated genes in all mouse models of SSc, 


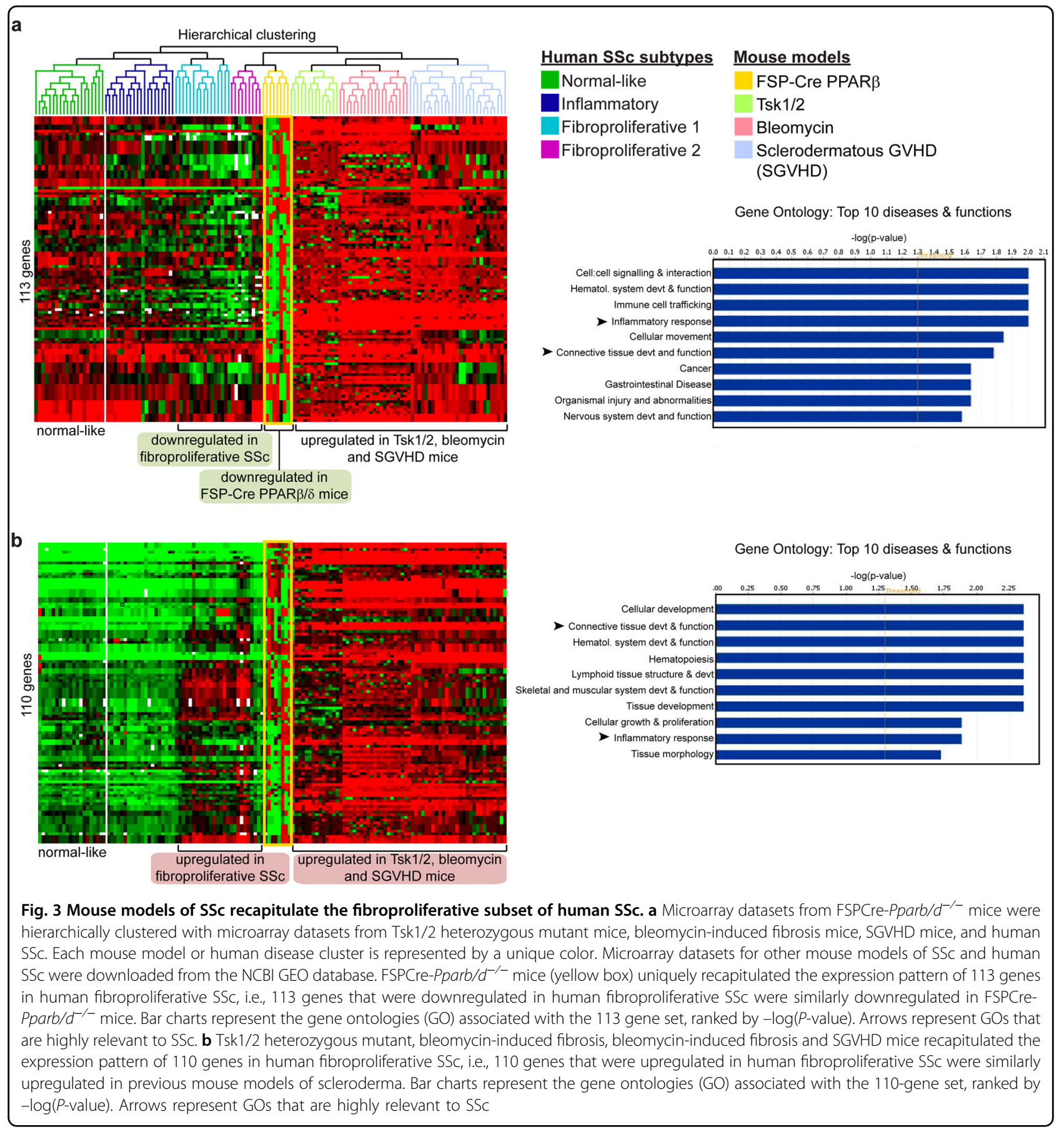

including our FSPCre-Pparb/d $d^{-1-}$ mice, highlight connective tissue abnormalities and inflammation as critical processes in the development of SSc.

Because our FSPCre-Pparb/d $d^{-1-}$ mice uniquely recapitulated the cell:cell signaling aspect of fibroproliferative 2 subtype of human SSc, we separated the epidermis and the dermis of FSPCre-Pparb/d ${ }^{-1-}$ mice skin to analyze their compartment-specific gene expression by microarray. Genes that were differentially regulated by $>1.5$ - fold in the epidermis of FSPCre-Pparb/ $/ d^{-1-}$ mice enriched the GO of lipid metabolism, molecular transport, small molecule biochemistry, cell death and survival, and cellular movement, ranked according to their $P$-values (a lower $P$-value indicates a stronger association to the indicated GO) (Fig. 4a). A total of 80 unique gene transcripts were responsible for the top three enriched GOs in the epidermis. Among these 80 genes, $85 \%$ of them (68 genes: 37 upregulated, 31 downregulated) were lipid- 

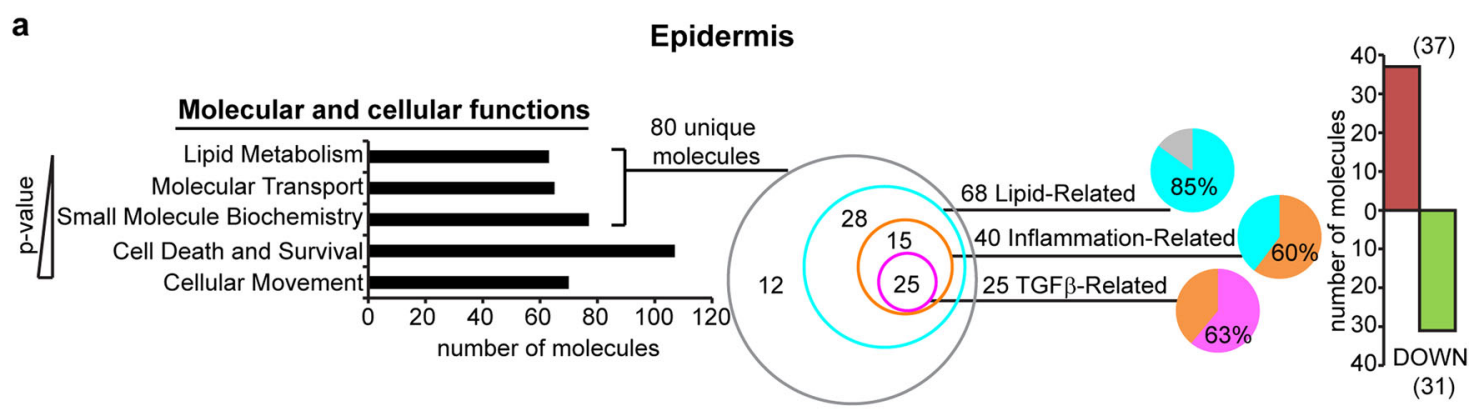

b

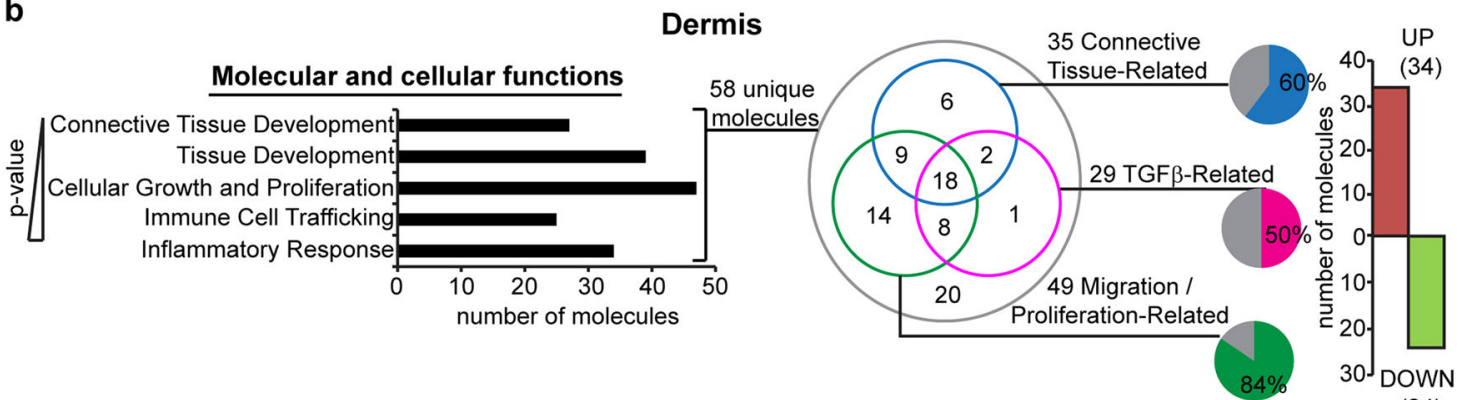

(24)

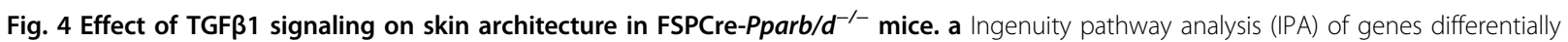
expressed by $>1.5$-fold in FSPCre-Pparb/d ${ }^{-/-}$epidermis relative to Pparb/d/f/f epidermis. Top 5 gene ontologies (GO) $(P<0.0341)$ and their numbers of constituent molecules were identified. Lower $P$-values indicate stronger associations with the indicated $\mathrm{GO}$. Further stratification of the 80 unique molecules identified lipid-related, inflammation-related, and TGF $\beta$-related sub-ontologies is shown. The bar chart represents the number of genes upregulated (red) or downregulated (green) by $>1.5$-fold in FSPCre-Pparb/d ${ }^{-/}$epidermis compared to Pparb/d/f/f epidermis. b IPA of genes differentially expressed by $>1.5$-fold in FSPCre-Pparb/ $/ d^{-1}$ dermis relative to Pparb/d/fl dermis. Top $5 \mathrm{GO}(P<0.0197)$ and their numbers of constituent molecules were identified. Lower $P$-values indicate stronger associations with the indicated GO. Further stratification of the 58 unique molecules identified connective tissue-related, migration/proliferation-related, and TGF $\beta$-related sub-ontologies. The bar chart represents the number of genes upregulated (red) or downregulated (green) by $>1.5$-fold in FSPCre-Pparb/d $d^{-/-}$dermis compared to Pparb/d/f/f dermis

related. 40 of these 68 genes $(\sim 60 \%)$ had overlapping functions in inflammation, and 25 of these 40 inflammation-related genes $(\sim 63 \%)$ were involved in TGF $\beta 1$ signaling (Fig. 4a, S4a). In the dermis, 58 genes that were differentially regulated by $>1.5$-fold, enriched the GOs of connective tissue development, tissue development, cellular growth and proliferation, immune cell trafficking and inflammatory response. 49 of the 58 genes $(\sim 85 \%)$ were migration/proliferation-related, 35 genes ( $\sim 60 \%)$ were connective tissue-related and 29 genes (50\%) were involved in TGF $\beta 1$ signaling (Fig. 4b, S4b).

The findings from microarray analysis of the two skin compartments suggest that TGF $\beta 1$ signaling is a major paracrine axis that is altered by the deletion of PPAR $\beta / \delta$ in fibroblasts. The microarray data is consistent with the increased immune cell infiltration within the skin of FSPCre-Pparb/d $d^{-1-}$ mice compared with their wild-type counterparts. This is also consistent with our current observation of epidermal hyperproliferation in the skin of FSPCre-Pparb/d $d^{-1-}$ mice. These observations suggest that the deletion of $\operatorname{PPAR} \beta / \delta$ in fibroblasts creates a hotbed for facilitated immune cell entry, which is in part responsible for thickening of the skin.

\section{Fibroblast PPAR $\beta / \delta$ regulates $\operatorname{Lrg} 1$ to modulate TGF $\beta 1$ signaling}

The interrogation of our microarray datasets suggested that dysregulated TGF $\beta 1$ signaling plays a central role in the profibrotic phenotype of FSPCre-Pparb/d $d^{-1-}$ mice (Fig. S4). Indeed, FSPCre-Pparb/d $/{ }^{-1-}$ mice exhibited an enhanced activation of TGF $\beta$ RII and SMAD3 via phosphorylation compared to Pparb/ $d^{\mathrm{fl} / \mathrm{fl}}$ mice (Fig. 5a). Of interest is the diminished expression of LRG1 in the dermis of FSPCre-Pparb/d $d^{-1-}$ mice (Fig. 5b, S4b). LRG1 modulates TGF $\beta 1$ signaling in a tissue-specific manner and has been implicated to play a role in vasculariza$\operatorname{tion}^{36,37}$. Thus, the role of LRG1 is aligned with the dysfunctional microvascularization and dysregulated TGF $\beta 1$ signaling observed in SSc patients. PPAR $\beta / \delta$ agonist GW501516 (GW) increased LRG1 mRNA and protein levels, which were attenuated either by $\mathrm{PPAR} \beta / \delta$ antagonist GSK0660 (GSK) or an inverse agonist compound $10 \mathrm{~h}(\mathbf{1 0 h})$ in normal primary human fibroblasts (Fig. 5c, d). In silico analysis of the intragenic regulatory region of mouse and human Lrg1 promoters revealed putative PPAR response elements (PPREs) (Fig. S5). Chromatin immunoprecipitation of primary mouse and 


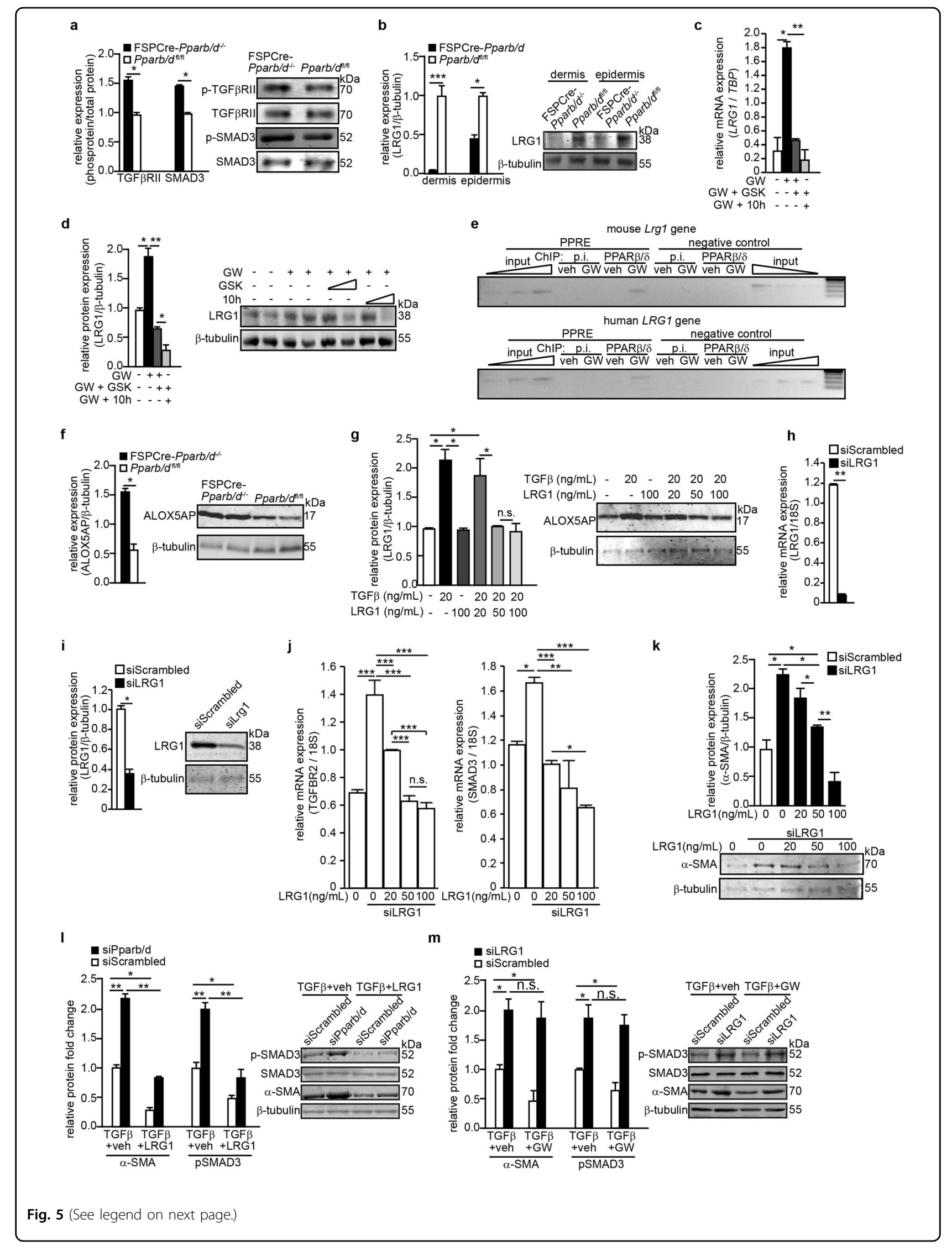


(see figure on previous page)

Fig. 5 Fibroblast PPAR $\beta / \delta$ modulates TGF $\beta 1$ signaling via LRG1. a, b Relative expression levels of phosphorylated TGF $\beta R \|$ and SMAD3 (a) and LRG1 proteins in dermis and epidermis (b) from FSPCre-Pparb/d ${ }^{-1}$ and Pparb/ ${ }^{f / / 7}$ mice. Representative immunoblots for phosphorylated, total TGFBRII and SMAD3, as well as LRG1 were shown. $\beta$-tubulin served as housekeeping protein was from the same samples. Values are mean \pm S.D. $(n=5)$. c, d Relative LRG1 mRNA (c) and protein (d) levels in human fibroblasts treated with GW501516 (GW), a specific PPARß/ $\delta$ agonist, and in combination with GSK0660 (GSK), a PPAR $/ \delta$ antagonist, and compound 10h (10h), a PPAR $/ \delta$ inverse agonist. TBP, TATA-Box binding protein, served as housekeeping gene for qPCR. Representative immunoblots are shown. $\beta$-tubulin that served as housekeeping protein was from the same samples. Values are mean \pm S.D. $(n=5)$. e Chromatin immunoprecipitation (ChIP) using PPAR $/ \delta$ antibodies or pre-immune Ig (p.i.) in human and mouse fibroblasts that were stimulated with either vehicle (Veh) or GW. Enrichment of either a PPRE-containing fragment from the endogenous $\mathrm{Lrg} 1$ promoter or a control DNA fragment ( $2 \mathrm{~kb}$ upstream of the PPRE) was evaluated by PCR. Aliquots of the extract were also analyzed before immunoprecipitation (input). $\mathbf{f}, \mathbf{g}$ Relative ALOX5AP protein level in skins from FSPCre-Pparb/d $/-$ and Pparb/d//fl mice (f) and in human keratinocytes treated with either TGF $\beta 1$ or recombinant LRG1 proteins $(\mathbf{g})$. Representative immunoblots for ALOX5AP were shown. $\beta$-tubulin served as

housekeeping protein was from the same samples. Values represent mean \pm S.D. $(n=5)$. h, i Relative LRG1 mRNA (h) and protein (i) levels in human fibroblasts transfected with either siRNA against LRG1 or scrambled siRNA control. $18 \mathrm{~S}$ rRNA served as housekeeping gene for qPCR. Representative immunoblot for LRG1 is shown. $\beta$-tubulin that served as housekeeping protein was from the same samples. Values are mean \pm S.D. $(n=3)$. j, $\mathbf{k}$ Relative expression of TGFBR2, SMAD3 mRNAs (j) and a-SMA protein (k) in control, LRG1-knockdown human fibroblasts, and LRG1-knockdown human fibroblasts treated with an increasing dose of recombinant LRG1 protein. 18S rRNA served as housekeeping gene for qPCR. Representative immunoblot for a-SMA was shown. $\beta$-tubulin that served as housekeeping protein was from the same samples. Values are mean $\pm S$.D. $(n=3)$. I, $\mathbf{m}$

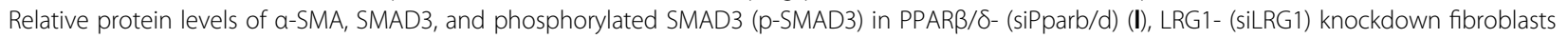
(m) and control fibroblasts transfected with scrambled siRNA (siScrambled). p-SMAD3 was normalized to total SMAD3 protein. $\beta$-tubulin that served as housekeeping protein was from the same samples. Values are mean \pm S.D. $(n=3) .{ }^{*} P<0.05 ;{ }^{* *} P<0.01 ;{ }^{* *} P<0.001 ;$ n.s. not significant

human fibroblasts showed that PPAR $\beta / \delta$ is recruited to the PPRE of $\operatorname{Lrg1}$ (Fig. 5e). These experiments identified Lrg1 as a direct target of PPAR $\beta / \delta$ in fibroblasts.

Microarray analyses revealed that the expression of genes related to lipid mediators of inflammation was upregulated in FSPCre-Pparb/d -1- $^{-}$epidermis (Fig. 4a). One of the most upregulated genes was arachidonate 5lipoxygenase-activating protein (Alox5ap), which mediates inflammatory responses towards external stimuli ${ }^{38}$ (Fig. S4). The FSPCre-Pparb/d $/ d^{-1-}$ mice expressed higher levels of ALOX5AP mRNA and protein in the epidermis than Pparb/d $f^{f l f l}$ mice (Fig. 5f). Recombinant LRG1 decreased ALOX5AP expression in a dose-dependent manner in primary human keratinocytes (Fig. 5g). These results also suggest that the expression of ALOX5AP in the epidermis was regulated, in part, by paracrine production of LRG1 by the fibroblasts. We conclude that the upregulation of ALOX5AP in the epidermis of FSPCrePparb $/ d^{-1-}$ mice may contribute to the increased susceptibility of the epidermis to inflammatory insults.

The contractile and secretory $\alpha$-SMA-positive myofibroblasts contribute to tissue repair during wound healing, but organ function can be severely impaired when contraction and collagen secretion become excessive, such as in fibrosis. ${ }^{3,39}$ We studied the TGF $\beta 1$-mediated $\alpha$ SMA expression in LRG1-deficient fibroblasts (Fig. 5h, i). LRG1 deficiency led to an increase in the mRNA levels of TGF $\beta$ RII and SMAD3, whereas treatment of LRG1deficient fibroblasts with recombinant LRG1 reduced TGF $\beta$ RII and SMAD3 expression (Fig. 5j). The increased expression of $\alpha$-SMA observed in LRG1-deficient fibroblasts was indicative of enhanced fibroblast activation
(Fig. 5k), consistent with the higher number of $\alpha$-SMApositive fibroblasts in FSPCre-Pparb/d ${ }^{-1-}$ mice. The exposure to increasing doses of recombinant LRG1 protein decreased the expression of $\alpha$-SMA in a dosedependent manner (Fig. 5k). To examine if recombinant LRG1 protein could rescue the fibrotic phenotype of PPAR $\beta / \delta$-deficient fibroblasts, we treated control (siScrambled) and PPAR $\beta / \delta$-knockdown (siPPARb/d) human fibroblasts with TGF $\beta 1$ in the presence or absence of recombinant LRG1 protein (Fig. 5l). TGF $\beta 1$ treatment resulted in higher expression of phosphorylated SMAD3 (p-SMAD3) and $\alpha$-SMA in siPPARb/d fibroblasts when compared with siScrambled fibroblasts (Fig. 5l). Importantly, this increase expression of p-SMAD3 and $\alpha$-SMA was attenuated in the presence of recombinant LRG1. Conversely, we treated siScrambled-knockdown and LRG1-knockdown (siLRG1) human fibroblasts with TGF $\beta 1$ in the presence or absence of PPAR $\beta / \delta$ agonist GW501516 (GW) (Fig. 5m). Similar to observation in Fig. 5k, reduced LRG1 resulted in higher p-SMAD3 and $\alpha$-SMA, i.e., greater fibroblast activation in siLRG1 fibroblasts when compared with control. The cotreatment with GW did not significantly attenuate the increase (Fig. 5m). Thus, LRG1 acted downstream of PPAR $\beta / \delta$ and was able to rescue the fibrotic phenotype of PPAR $\beta / \delta$-deficient fibroblasts.

These observations suggest a role of fibroblast PPAR $\beta / \delta$ in the homotypic regulation of TGF $\beta 1$ signaling, via increased LRG1 production, in fibroblast activation and collagen production, which contribute to the fibrotic phenotype of FSPCre-Pparb/d $/ d^{-1}$ mice. Taken together, our results indicate that in fibroblasts, PPAR $\beta / \delta$ 


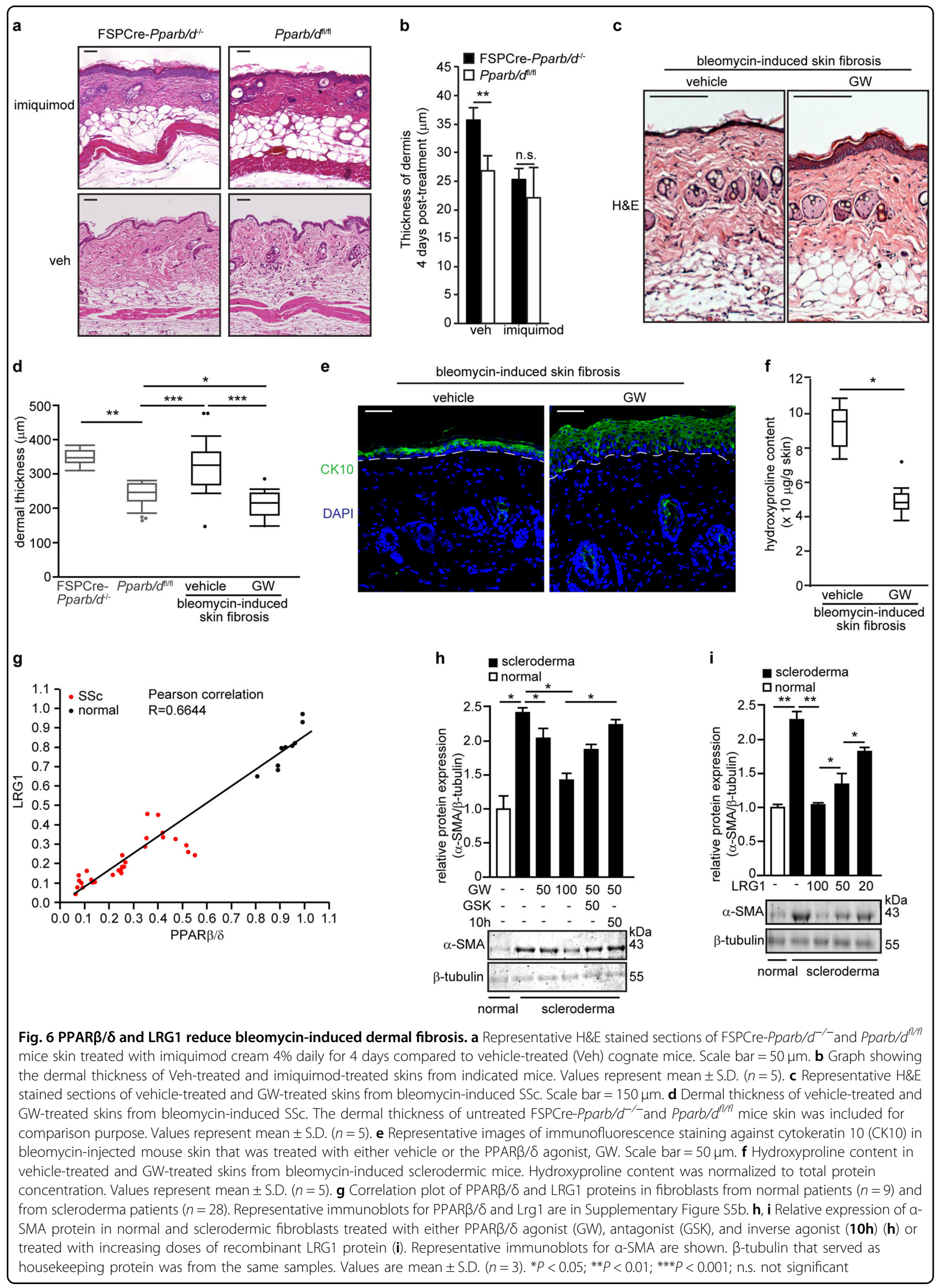


transcriptionally upregulates $\operatorname{Lrg} 1$ that in turn acts in an autocrine and paracrine manner to modulate tissuespecific TGF $\beta 1$ effects.

Ligand-activated PPAR $\beta / \delta$ reduces the fibrotic effect in SSc

Although the best treatment for localized SSc is uncertain and current treatments often fail to help, benefits have been reported following topical $5 \%$ imiquimod cream (Aldara ${ }^{\circ}$ ) treatment ${ }^{40-43}$. Imiquimod is an immune response modifier. It induces interferon and subsequently inhibits TGF $\beta 1$ and was reported to attenuate SSc symptoms in patients ${ }^{40}$. FSPCre-Pparb/d $d^{-1-}$ mice topically treated with imiquimod exhibited a thinning of the thickened dermis (Fig. 6a). The resultant dermal thickness was no longer significantly different from $P p a r b / d^{f l / f l}$ (Fig. 6b). To evaluate the potential of PPAR $\beta / \delta$ as a therapeutic target for dermal fibrosis, we examined the effect of the PPAR $\beta / \delta$ agonist GW on the widely used bleomycin-induced mouse model of dermal fibrosis. Dermal fibrosis was induced in $\mathrm{C} 3 \mathrm{H} / \mathrm{He}$ wildtype mice through daily subcutaneous injection of bleomycin for a period of 28 days. We concurrently co-injected them with either dimethyl sulfoxide (DMSO) (vehicle) or GW. Histological analysis revealed that the dermis of the mice cotreated with GW were thinner than that of mice cotreated with vehicle (Fig. 6c, d). The epidermis of mice cotreated with GW showed increased differentiation of the epidermal layer by PPAR $\beta / \delta$ activation, as evidenced by an increased CK10 staining (Fig. 6e). Hydroxyproline assay also showed a lower level of collagen in the dermis of GW-treated mice (Fig. 6f).

To further underscore the clinical relevance of PPAR $\beta /$ $\delta$, we examined the effect of GW on human dermal fibroblasts that were explanted from 28 SSc patients. Scleroderma fibroblasts expressed lower levels of PPAR $\beta$ / $\delta$ mRNA and protein than normal fibroblasts (Fig. $6 \mathrm{~g}, \mathrm{~h}$ ). The protein level of LRG1 was lower in SSc fibroblasts than in normal fibroblasts isolated from healthy volunteers (Fig. 6h). GW-activated PPAR $\beta / \delta$ reduced the expression of $\alpha$-SMA in SSc fibroblasts, while the cotreatment with either GSK or compound 10h $(\mathrm{PPAR} \beta / \delta$ inverse agonist) increased the expression of $\alpha$-SMA (Fig. 6i). Recombinant LRG1 similarly reduced the expression of $\alpha$-SMA in SSc fibroblasts (Fig. 6j), indicating that PPAR $\beta / \delta$ exerted its effect through LRG1, a direct downstream target of PPAR $\beta / \delta$. This observation suggested that the activation of PPAR $\beta / \delta$ could reduce fibrosis, underscoring its therapeutic potential.

\section{Discussion}

Connective skin diseases are characterized by dermal fibrosis and excessive collagen deposition in the skin and internal organs. Fibroblasts are the main producers of collagen in the extracellular matrix, and thus the pivotal cell type involved in the clinical presentation of connective skin diseases, including SSc. Yet, the transcriptional networks within the fibroblasts that contribute to dermal fibrosis are not well-studied. We showed here that fibroblast PPAR $\beta / \delta$ is not critical for embryonic development in mice. However, the epidermis of FSPCrePparb/d - $^{-1}$ mice had an aggravated response to TPA, suggesting that $P P A R \beta / \delta$ ablation in the fibroblasts rendered the mutant mice more susceptible to developing inflammatory skin disorders. Notably, FSPCre-Pparb/d ${ }^{-1}$ - mice exhibited pronounced dermal thickening associated with excessive collagen production, a greater number of myofibroblasts and Ly6-positive immune cells, which somewhat represents a fibrosing skin disorder phenotype. These observations were also supported by our comparative gene analyses, which showed that the expression of the respective genes for collagen production and immune cells activation were correspondingly altered.

The FSPCre-Pparb/ $/ d^{-1-}$ mice exhibited dermal fibrosis. The skin of FSPCre-Pparb/d $d^{-1-}$ mice was characterized by increased collagen synthesis associated with increased activation of fibroblasts to myofibroblasts. However, FSPCre-Pparb/d $/ d^{-1-}$ livers exhibited neither hepatomegaly, which manifests with systemic SSc-associated primary biliary cirrhosis ${ }^{44}$, nor increased collagen deposition in the intestinal wall, which could cause bowel hypomobility $^{45}$. These indicate that the role of PPAR $\beta / \delta$ in fibroblasts is likely to be tissue/organ-specific. We detected dysregulated TGF $\beta 1$ signaling in the FSPCrePparb/d $d^{-1-}$ mice, which has been implicated in the etiology of dermal fibrosis ${ }^{46}$. Dermal fibrotic symptoms may be further aggravated by an enhanced epidermal inflammatory response in these mutant mice. In clinical settings, activated fibroblasts give rise to an immune-inflammatory environment with abnormal repair of damaged vessels and fibrosis ${ }^{47,48}$ and ROS production $^{49-52}$. PPAR $\beta / \delta$-deficient fibroblasts contribute to greater microenvironment oxidative stress due to reduced expression of antioxidant genes such as catalase and glutathione peroxidase $1^{26}$. Microarray analyses of FSPCre-Pparb/d $/ d^{-1}$ skin revealed upregulation of numerous genes that would culminate an increased ROS production in the PPAR $\beta / \delta$-ablated dermis, including CCL2 and CCL5. Notably, CCL2 and CCL5 upregulation was found to precede skin and lung fibrosis in a mouse model that recapitulates the pathology of $\mathrm{SSc}^{53}$. Finally, we also identified gene networks in FSPCre-Pparb/d - $^{-1}$ mice that closely resemble fibroproliferative 2 subtype of human SSc. Thus, the fibroblast $\mathrm{PPAR} \beta / \delta$ deficiency causes dermal fibrosis. Unlike FSPCre-Pparb/d ${ }^{-1-}$ mice, fibroblast-specific PPAR $\gamma$-deficient skin did not exhibit any manifestations of dermal fibrosis or collagen overproduction, suggesting that fibroblast $\mathrm{PPAR} \beta / \delta$ and PPAR $\gamma$ play different roles in skin physiology ${ }^{14}$. FSPCre- 
Pparb/d $d^{-1-}$ exhibited epidermal hyperplasia which is characteristic of psoriasis, another type of connective tissue diseases. Several studies have also implicated a role for PPAR $\beta / \delta$ in psoriasis ${ }^{54-58}$. However, the relative contribution of epidermal and dermal PPAR $\beta / \delta$ in the etiology and pathophysiology of psoriasis remains unclear. This study is still ongoing in the lab, and is beyond the scope of this work.

Our current study revealed that TGF $\beta 1$ signaling has an overarching influence on skin architecture in FSPCrePparb/d ${ }^{-1-}$ mice. We identified Lrg1 as a direct downstream target of PPAR $\beta / \delta$ that contributes to the observed phenotype. Thus far, LRG1 had been reported to augment endothelial TGF $\beta 1$ signaling and promote angiogenesis ${ }^{36}$. LRG1 is expressed in various human tissues, including the breast, skin, and intestine, but little is known about its role in these tissues. Our study shows that fibroblastsecreted LRG1 acts in a paracrine manner to influence the epidermal TGF $\beta 1$ response to inflammatory challenges. The underlying mechanism involved transcriptional upregulation of LRG1 by fibroblast $\mathrm{PPAR} \beta / \delta$, which modulated homotypic and heterotypic TGF $\beta 1$ signaling. Limitations of our study include that one genetic background of mouse and our deletion strategy consists of the deletion of exons coding for the DNA-binding domain of $\operatorname{PPAR} \beta / \delta$. It is also conceivable that other gene deletion strategies may result in different phenotypic severity or outcomes $^{8}$. In addition to fibroblasts, FSP1 driven gene deletion may affect other cell types, although in vitro experiments were performed to strengthen the conclusion. Nevertheless, to the best of our knowledge, this is the first study to examine the role of fibroblast PPAR $\beta / \delta$ in skin, which reveals novel epithelial-mesenchymal communication.

Current treatment of SSc is directed toward managing complications and providing symptomatic relief. Although several regimens have shown benefit in case series, few controlled trials have been performed and data suggest wide variation in the approach to treatment ${ }^{59}$. For example, topical 5\% imiquimod cream has been reported for the treatment of localized $\mathrm{SSc}^{40}$. We showed that FSPCre-Pparb/d $d^{-1-}$ mice were responsive to topical treatment with imiquimod cream. The dermis of these mice became thinner, resulting in a thickness similar to that of their wildtype littermates. We also showed that ligand-activated PPAR $\beta / \delta$ reduced dermal fibrosis using the bleomycin-induced mouse model of dermal fibrosis. In the 28 SSc patients analyzed, fibroblasts consistently had reduced level of PPAR $\beta / \delta$ and LRG1 compared to healthy subjects. In addition, PPAR $\beta / \delta$ activation, or LRG1 treatment, could reduce $\alpha$-SMA levels in these profibrotic fibroblasts. In conclusion, we identified an important signaling conduit that integrates PPAR $\beta / \delta$ and
TGF $\beta 1$ signaling networks via LRG1 to be key players in fibrosis.

\section{Materials and methods \\ Cell culture}

Human keratinocytes (Lonza, USA) were routinely cultured in keratinocyte-SFM culture media (Invitrogen, USA). Fibroblasts were maintained in FibroGRO ${ }^{\mathrm{TM}}$ Medium (Millipore, USA). All cultures were maintained in a humidified incubator at $37^{\circ} \mathrm{C}$ with $5 \% \mathrm{CO}_{2}$. Normal human dermal and sclerodermic fibroblasts were procured from Asterand Bioscience, UK and Proteogenex, USA. Scleroderma patients were classified accordingly to American College of Rheumatology criteria ${ }^{60}$. SSc patients presented limited cutaneous SSc with skin thickening was limited to hands and face. SSc patients have Raynaud's phenomenon for $>5$ years, minimal joint pain and presented telangiectasias. The male:female ratio was approximately 2:7. The balanced median age of the normal $(n=9)$ and SSc $(n=28)$ donors were $42.8 \pm 3.1$ and $49.1 \pm 2.5$ years old, respectively. Due to the role of $\mathrm{PPAR} \beta / \delta$ in energy homeostasis, patients with diabetes, obese patients (BMI > 30) or patients with hyperlipemia were excluded. Skin fibroblasts were isolated from skin biopsy of the forearm using the explant method ${ }^{61}$ and flash frozen at the third passages for subsequent analysis.

\section{Antibodies and chemicals}

Primary antibodies used in this study: monoclonal anti$\alpha$-SMA, PCNA, GAPDH, polyclonal anti-PPAR $\beta / \delta, \beta-$ tubulin, TGase1, collagen type I and ALOX5AP (Santa Cruz, USA); polyclonal anti-Ly6C/Ly-6G (BioLegend, USA); polyclonal anti-vimentin and LRG1 (Abcam, USA). IRDye 680LT conjugated secondary antibodies were from Li-Cor (Biosciences, USA); DyLight conjugated secondary antibodies were from Vector Laboratories, USA. GW501516 (PPAR $\beta / \delta$ agonist; GW), GSK0660 (PPAR $\beta / \delta$ antagonist; GSK) and compound 10h (PPAR $\beta / \delta$ inverse agonist; 10h) were synthesized in-house.

\section{Generation of fibroblast-selective PPAR $\beta / \delta$-knockout mice}

The $P P A R b / d^{f l f l}$ mouse was provided by Prof Walter Wahli (University of Lausanne, Switzerland). The PPARb/ $d^{f l f l}$ mouse carries alleles of the $P P A R \beta / \delta$ gene where the exon 4, which encodes the DNA-binding domain, was flanked by loxP sites ${ }^{62,63}$. The FSPCre transgenic mouse expresses Cre recombinase under the control of the FSP1 promoter $^{64}$. FSPCre mice were always maintained as hemizygotes. FSPCre and PPARb/d $d^{l l f l}$ mice were independently backcrossed to C57BL/6J wild-type mice for at least six generations. The FSPCre-Pparb/d $d^{+/-}$mouse was generated in-house by crossing PPARb/dl/fl and FSPCre mice. FSPCre-Pparb/d $d^{+/-}$progenies were bred with 
Pparb $/ d^{\mathrm{fl} / \mathrm{fl}}$ mice to obtain FSPCre-Pparb/d $d^{-1-}$ mice and the wild-type control mice. To sustain this genotype, we bred FSPCre-Pparb/ $/ d^{-1-}$ progenies with Pparb/d $d^{\mathrm{f} / \mathrm{fl}}$ mice. Mice were housed in a specific-pathogen free facility with a $12 \mathrm{~h} / 12 \mathrm{~h} \mathrm{light/dark}$ cycle. Food and water were provided ad libitum.

Mice in the telogen phase of their hair cycle were anaesthetized and depilated on their dorsal back before being subjected to a biopsy punch $(\sim 8 \mathrm{~mm})$. The skin biopsy was then treated with $3.8 \%$ ammonium thiocyanate (Sigma, USA) in $1 \times \mathrm{PBS}$ for $30 \mathrm{~min}$ at $37^{\circ} \mathrm{C}$. Subsequently, the epidermis was mechanically separated from the dermis with a pair of forceps. The dermis was then curetted with a 30-gauge needle. The dermis was washed with $1 \times$ PBS before being subjected to the genotyping procedure, real-time qPCR assay and Western blot. Mice genotyping were performed using the Mouse Genotyping Kit HotStart as described by manufacturer (KAPA Biosystems, USA). Genotyping primers are provided in Table S1.

Animal experiments were carried out in accordance to the guidelines of the University Institutional Animal Care and Use Committee (IACUC, ARF-SBS/NIE-A0216AZ, A0321, and A0322), Singapore.

\section{Real-time qPCR and Western blot}

RNA extraction, reverse transcription, qPCR and western blot were performed as previously described ${ }^{65}$. Total RNA was extracted using TRIzol ${ }^{\circ}$ as described by manufacturer (Invitrogen, USA). Reverse transcription was performed using the iScript ${ }^{\mathrm{TM}}$ Reverse Transcription Supermix for RT-qPCR (Bio-Rad, USA) and qPCR done using KAPA SYBR ${ }^{\circ}$ FAST Universal qPCR Kit (KAPA Biosystems, USA). Primer sequences are provided in Table S1. Total protein was extracted using M-PER ${ }^{\mathrm{TM}}$ Mammalian Protein Extraction Reagent (ThermoFisher Scientific). Infrared western blot was done as previously described $^{66}$. Protein bands were analyzed using the Odyssey CLx scanner and Image Studio V2.1 (LI-COR Biosciences, USA).

\section{Histological, immunohistochemical, and immunofluorescence staining}

Tissues were processed, sectioned and stained as previously described ${ }^{65}$. PicroSirius Red staining was performed as recommended by the manufacturer (ab150681, Abcam, Cambridge, United Kingdom). Colorimetric images were captured using Axio Scan.Z1 with Plan Apochromat $20 \times / 0.8$ objective (Carl Zeiss, Germany). Fluorescence images were captured using the LSM 710 confocal microscope with the Zeiss EC Plan-NEOFLUAR $20 \times / 0.5$ NA objective. Analyses were performed with ZEN 2012 Light Edition software (Carl Zeiss, Germany).

\section{Lucifer yellow permeability assay}

Lucifer yellow dye ( $1 \mathrm{mg} / \mathrm{mL}$; Sigma-Aldrich, USA) was topically applied on the epidermis for $1 \mathrm{~h}$. Excess Lucifer yellow was washed away with $1 \times \mathrm{PBS}$, and the skin tissues were cryofrozen with OCT tissue freezing medium (Leica, USA). Tissues were cryosectioned at $8 \mu \mathrm{m}$ and mounted with Hoechst 33,342 dye (Life Technologies, USA). Images were taken using the LSM 710 confocal microscope with the Zeiss EC Plan-NEOFLUAR $20 \times / 0.5$ NA objective. Analyses were performed with ZEN 2012 Light Edition software (Carl Zeiss, Germany).

\section{Hydroxyproline assay}

Hydroxyproline content in tissues was determined as previously described ${ }^{67}$. Trans-4-hydroxy-L-proline $(0-300 \mu \mathrm{g} / \mathrm{mL})$ was used to generate a standard graph. Absorbance was measured at $550 \mathrm{~nm}$ using a SpectraMax M2e Multi-Mode Microplate Reader and SoftMax Pro Microplate Data Acquisition \& Analysis Software (Molecular Devices, USA). The value of unknown hydroxyproline was determined from the standard graph. The weight of each respective wound biopsy was used for normalization.

\section{2-O-tetradecanoylphorbol 13-acetate (TPA)-induced skin inflammation}

Mice of $\sim 4$ weeks of age were shaven on their dorsal skin and topically treated with $6.5 \mathrm{nmol}$ of TPA (SigmaAldrich, USA) in acetone for $24 \mathrm{~h}$. The vehicle-treated and TPA-treated skins were then harvested and processed accordingly for histological studies and protein analysis.

\section{Wounding experiment}

Mice of $\sim 8$ weeks of age were shaved and full-thickness excisional wounds were inflicted on the dorsal skin of mice as previous described ${ }^{68}$. Wound surface areas and contraction rates were measured over the indicated duration.

\section{Imiquimod topical application}

Mice of $\sim 8$ weeks of age were shaved and subsequently imiquimod cream $4 \%$ (Selleck Chemicals, USA) was applied topically daily for a period of 4 days. Skin tissues were then harvested and processed for histological analysis.

\section{Bleomycin-induced dermal fibrosis}

$\mathrm{C} 3 \mathrm{H} / \mathrm{He}$ mice of 4 weeks of age were subcutaneously injected with $10 \mu \mathrm{g}$ of bleomycin sulfate (Selleck Chemicals, USA) together with either DMSO $(n=5)$ or $0.25 \mathrm{mg}$ of GW $(n=5)$ daily for a period 28 days ${ }^{69}$. Skin tissues were then harvested and processed accordingly for histological studies. 


\section{Gene expression microarray}

cDNA synthesis was performed using the Applause WT-Amp ST System (NuGEN Technologies, USA). The resulting cDNA was purified with MinElute Reaction Cleanup Kit (Qiagen, USA), followed by fragmentation and labeling using the Encore Biotin Module (NuGEN Technologies, USA). The fragmented and labeled cDNA were mixed with a hybridization master mix (Affymetrix, USA) in accordance to the manufacturer's protocol, and $90 \mu \mathrm{L}$ of the resultant mixture were injected into the GeneChip Mouse Gene 1.0 ST Array gene chips (Affymetrix, USA). The scanned data was collected for analyses using the Partek Genomics Suite v6.6 (Partek Inc., USA). Differences in gene expression between FSPCre-Pparb/ $d^{-/-}$and wild-type samples were identified using the ANOVA function of the Partek Genomics Suite. Microarray datasets from FSPCre-Pparb/d $d^{-/}$mice were subsequently hierarchically clustered with microarray datasets from tight skin (Tsk) 1 or 2 heterozygous mutant mice (GSE71999), bleomycin-induced fibrosis mice (GSE71999), sclerodermatous graft versus host disease (SGVHD) mice (GSE24410), and human samples of SSc (PMC2481301, GSE9285), downloaded from NCBI Gene Expression Omnibus (GEO) database. We focused our analysis on an intrinsic SSc gene set comprising 995 genes that was previously reported ${ }^{33}$ (GSE9285). Gene ontology (GO) analysis of gene subsets was performed using the Ingenuity Pathway Analysis software (Ingenuity Systems Inc., USA). The microarray protocols and data have been deposited in NCBI's Gene Expression Omnibus (GEO) database accession number GSE71419.

\section{Chromatin immunoprecipitation (ChIP)}

ChIP experiments were performed as previously described $^{65}$ except that anti-PPAR $\beta / \delta$ antibodies was used (see above). ChIP primer sequences are listed in Table S1.

\section{Transfection and luciferase assay}

TGF $\beta 1$ responsive luciferase reporter assay in keratinocytes was performed as previously described ${ }^{70}$. Luciferase assay was performed using the Dual Luciferase Reporter Assay System and the Glomax 20/20 Luminometer (Promega, USA). The ON-TARGETplus SMARTpool siRNA were used for knockdown studies (Lrg1, Cat \# L-056177-01; PPAR $\beta / \delta$, Cat \#L-042751-01; Dharmacon). Cells were transfected using DharmaFect 1 as recommended by manufacturer. As control, the ONTARGETplus non-targeting control pool (D-001810-10; Dharmacon) was used.

\section{Anti-nucleosome antibody (ANA) detection}

Mice of 4-6 weeks of age were studied for their ANA levels. ANA levels in mice plasma samples were measured by using the anti nucleosome IgG Antibody (AnuA, IgG)
BioAssay $^{\text {TM }}$ ELISA Kit (United States Biological, USA). Readings were performed on the Infinite ${ }^{\circ}$ M200 Pro machine (Tecan, Switzerland) with the Magellan ${ }^{\mathrm{TM}}$ v7.0 software (Tecan, Switzerland).

\section{Statistical analysis}

Statistical analyses were performed using two-tailed Mann-Whitney U test using SPSS software (IBM Corporation, USA). A $P$-value of $<0.05$ was considered significant.

\begin{abstract}
Acknowledgements
This work is supported by the Singapore Ministry of Education under its Singapore Ministry of Academic Research Fund Tier 2 (MOE2010-T2- 2-009) to NST; (MOE2012-T2-1-014) to SC, (MOE2014-T2-1-036) to XW and WW, (MOE2015-T1-001-034) to WW and NST; and A*STAR-NHG-NTU Skin Research Grant (SRG/14003) to NST; Lee Kong Chian School of Medicine, Nanyang Technological University (NTU) Start-Up Grant, the Région Midi-Pyrénées through the Chaire d'Excellence Pierre de Fermat and the Bonizzi-ThelerStiftung to WW. The authors are thankful to the Director, CSIR-NEIST, Jorhat for his support and SERB-Department of Science \& Technology (SERB-DST), Government of India for providing the Ramanujan Fellowship (SB/S2/RJN-087/ 2014) to Dr. Mintu Pal. We wish to thank Pierre Chambon and Daniel Metzger for their collaboration in generating the floxed PPAR $\beta / \delta$ mice. The authors also acknowledged the IMB-IMCB Joint Electron Microscopy Suite for assistance in preparing and imaging specimens for this work.
\end{abstract}

\section{Author details}

${ }^{1}$ School of Biological Sciences, Nanyang Technological University, 60 Nanyang Drive, Singapore 637551, Singapore. 'Lee Kong Chian School of Medicine, Nanyang Technological University, Novena Campus, 11 Mandalay Road, Singapore 308232, Singapore. ${ }^{3}$ Department of Microbiology, Tumor and Cell Biology, Karolinska Institutet, Nobels väg 16, 17177 Stockholm, Sweden. ${ }^{4}$ Biological Sciences and Technology Division, CSIR-North East Institute of Science and Technology, Jorhat, Assam 785006, India. ${ }^{5}$ School of Physical and Mathematical Sciences, Nanyang Technological University, Singapore 637371, Singapore. ${ }^{6}$ Institute of Molecular and Cell Biology, Agency for Science Technology \& Research, 61 Biopolis Drive, Proteos, Singapore 138673, Singapore. 'Department of Cell Biology, Institute of Ophthalmology, University College London, London, UK. ${ }^{8}$ Singapore Eye Research Institute, Singapore 169856, Singapore. ${ }^{9}$ INRA ToxAlim, Chemin de Tournefeuille, Toulouse Cedex 3 UMR1331, France. ${ }^{10}$ Center for Integrative Genomics, University of Lausanne, Le Genopode, Lausanne, Switzerland. ${ }^{11}$ KK Research Centre, KK Women's and Children Hospital, 100 Bukit Timah Road, Singapore 229899, Singapore

\section{Author contributions}

M.K.S. and N.S.T. designed, performed, and organized most of the experiments; analyzed and interpreted the data; and co-wrote the manuscript, which was edited and read by all authors. M.K.S., J.S.K.C., and E.H.P.T. performed microarray analyses. J.S.K.C., Z.T., T.P., and N.J.N.K. assisted in performing experiments and interpretation of data. P.Z. performed picrosirus red staining and imaging. J.W. K.W. and E.H.P.T. performed the cell sorting, FACS analysis and multiplex genotyping. J.P.C., C.K.T., and W.W. designed and executed experiments with mouse livers and gastrointestinal tract. B.M.K.T., Y.L.T., and S.C. synthesized and provided the various ligands of PPARß/ $\delta$. X.W. provided insights on LRG1related experiments and M.P. worked on normal and scleroderma fibroblasts experiments. W.W. provided the floxed PPARß/S mice. N.S.T., M.K.S., W.W., and X.W. are involved scientific discussion. N.S.T., M.K.S., and J.S.K.C. co-wrote the manuscript.

\section{Conflict of interest}

The authors declare that they have no conflict of interest.

\section{Publisher's note}

Springer Nature remains neutral with regard to jurisdictional claims in published maps and institutional affiliations. 
Supplementary Information accompanies the paper at (https://doi.org/ 10.1038/s41421-018-0014-5).

Received: 6 September 2017 Accepted: 4 January 2018 Published online: 03 April 2018

\section{References}

1. Hinchcliff, M. \& Varga, J. Systemic sclerosis/scleroderma: a treatable multisystem disease. Am. Fam Physician 78, 961-968 (2008).

2. Pattanaik, D., Brown, M. \& Postlethwaite, A. E. Vascular involvement in systemic sclerosis (scleroderma). J. Inflamm. Res. 4, 105-125 (2011).

3. Klingberg, F., Hinz, B. \& White, E. S. The myofibroblast matrix: implications for tissue repair and fibrosis. J. Pathol. 229, 298-309 (2013).

4. Young, A. \& Khanna, D. Systemic sclerosis: commonly asked questions by rheumatologists. J. Clin. Rheumatol. 21, 149-155 (2015).

5. Marangoni, R. G., Varga, J. \& Tourtellotte, W. G. Animal models of scleroderma: recent progress. Curr. Opin. Rheumatol. 28, 561-570 (2016).

6. Artlett, C. M. Animal models of scleroderma: fresh insights. Curr. Opin. Rheu matol. 22, 677-682 (2010).

7. Wu, M. \& Varga, J. In perspective: murine models of scleroderma. Curr. Rheumatol. Rep. 10, 173-182 (2008)

8. Tan, N. S., Vazquez-Carrera, M. \& Montagner, A. et al. Transcriptional control of physiological and pathological processes by the nuclear receptor PPARbeta/ delta. Prog. Lipid Res. 64, 98-122 (2016).

9. Schulman, I. G. Nuclear receptors as drug targets for metabolic disease. Adv. Drug Deliv. Rev. 62, 1307-1315 (2010).

10. Burris, T. P., Solt, L. A. \& Wang, Y. et al. Nuclear receptors and their selective pharmacologic modulators. Pharmacol. Rev. 65, 710-778 (2013).

11. Tan, N. S. \& Wahli, W. in Lipids and Skin Health (ed. Pappas, A.) 91-106 (Springer, Springer International Publishing AG, Cham (ZG) Switzerland, 2015).

12. Yin, K. \& Smith, A. G. Nuclear receptor function in skin health and disease: therapeutic opportunities in the orphan and adopted receptor classes. Cell. Mol. Life Sci. 73, 3789-3800 (2016).

13. Ruzehaji, N., Frantz, C. \& Ponsoye, M. et al. Pan PPAR agonist IVA337 is effective in prevention and treatment of experimental skin fibrosis. Ann. Rheum. Dis. 75, 2175-2183 (2016).

14. Sha, W., Thompson, K. \& South, J. et al. Loss of PPARy expression by fibroblasts enhances dermal wound closure. Fibrogenesis Tissue Repair 5, 5 (2012).

15. Kapoor, M., McCann, M. \& Liu, S. et al. Loss of peroxisome proliferator-activated receptor gamma in mouse fibroblasts results in increased susceptibility to bleomycin-induced skin fibrosis. Arthritis Rheum. 60, 2822-2829 (2009).

16. Tan, N. S., Icre, G. \& Montagner, A. et al. The nuclear hormone receptor peroxisome proliferator-activated receptor beta/delta potentiates cell chemotactism, polarization, and migration. Mol. Cell. Biol. 27, 7161-7175 (2007).

17. Tan, N. S., Michalik, L. \& Noy, N. et al. Critical roles of PPAR beta/delta in keratinocyte response to inflammation. Genes Dev. 15, 3263-3277 (2001).

18. Tan, N. S., Michalik, L. \& Di-Poi, N. et al. Critical roles of the nuclear receptor PPARbeta (peroxisome-proliferator-activated receptor beta) in skin wound healing. Biochem. Soc. Trans. 32, 97-102 (2004).

19. Di-Poï, N., Tan, N. S. \& Michalik, L. et al. Antiapoptotic role of PPARbeta in keratinocytes via transcriptional control of the Akt1 signaling pathway. Mol. Cell 10, 721-733 (2002).

20. Aden, N., Nuttall, A. \& Shiwen, X. et al. Epithelial cells promote fibroblast activation via IL-1alpha in systemic sclerosis. J. Invest. Dermatol. 130 2191-2200 (2010).

21. Suwara, M. I., Green, N. J. \& Borthwick, L. A. et al. IL-1alpha released from damaged epithelial cells is sufficient and essential to trigger inflammatory responses in human lung fibroblasts. Mucosal Immunol. 7, 684-693 (2014).

22. Chong, H. C., Tan, M. J. \& Philippe, V. et al. Regulation of epithelialmesenchymal IL-1 signaling by PPARbeta/delta is essential for skin homeostasis and wound healing. J. Cell Biol. 184, 817-831 (2009).

23. Gabrielli, A., Svegliati, S. \& Moroncini, G. et al. New insights into the role of oxidative stress in scleroderma fibrosis. Open Rheumatol. J. 6, 87-95 (2012).

24. Gabrielli, A., Svegliati, S. \& Moroncini, G. et al. Oxidative stress and the pathogenesis of scleroderma: the Murrell's hypothesis revisited. Semin. Immunopathol. 30, 329-337 (2008).

25. Sambo, P., Baroni, S. S. \& Luchetti, M. et al. Oxidative stress in scleroderma: maintenance of scleroderma fibroblast phenotype by the constitutive up- regulation of reactive oxygen species generation through the NADPH oxidase complex pathway. Arthritis Rheum. 44, 2653-2664 (2001).

26. Wang, X., Sng, M. K. \& Foo, S. et al. Early controlled release of peroxisome proliferator-activated receptor $\beta / \delta$ agonist GW501516 improves diabetic wound healing through redox modulation of wound microenvironment. $J$. Control. Release 197, 138-147 (2015).

27. Peters, J. M., Lee, S. S. \& Li, W. et al Growth, adipose, brain, and skin alterations resulting from targeted disruption of the mouse peroxisome proliferatoractivated receptor beta(delta). Mol. Cell. Biol. 20, 5119-5128 (2000).

28. Michalik, L., Desvergne, B. \& Tan, N. S. et al. Impaired skin wound healing in peroxisome proliferator-activated receptor (PPAR)alpha and PPARbeta mutant mice. J. Cell Biol. 154, 799-814 (2001).

29. Barak, Y., Liao, D. \& He, W. et al. Effects of peroxisome proliferator-activated receptor delta on placentation, adiposity, and colorectal cancer. Proc. Natl Acad. Sci. USA 99, 303-308 (2002).

30. Österreicher, C. H., Penz-Österreicher, M. \& Grivennikov, S. I. et al. Fibroblastspecific protein 1 identifies an inflammatory subpopulation of macrophages in the liver. Proc. Natl Acad. Sci. USA 108, 308-313 (2011).

31. Sato, S., Kodera, M. \& Hasegawa, M. et al. Antinucleosome antibody is a major autoantibody in localized scleroderma. Br. J. Dermatol. 151, 1182-1188 (2004).

32. Takehara, K. \& Sato, S. Localized scleroderma is an autoimmune disorder. Rheumatology 44, 274-279 (2005).

33. Milano, A., Pendergrass, S. A. \& Sargent, J. L. et al. Molecular subsets in the gene expression signatures of scleroderma skin. PLoS One 3, e2696 (2008).

34. Sargent, J. L., Li, Z. \& Aliprantis, A. O. et al. Identification of optimal mouse models of systemic sclerosis by interspecies comparative genomics. Arthritis Rheumatol. 68, 2003-2015 (2016).

35. Greenblatt, M. B., Sargent, J. L. \& Farina, G. et al. Interspecies comparison of human and murine scleroderma reveals $\mathrm{IL}-13$ and CCL2 as disease subsetspecific targets. Am. J. Pathol. 180, 1080-1094 (2012).

36. Wang, X., Abraham, S. \& McKenzie, J. A. G. et al. LRG1 promotes angiogenesis by modulating endothelial TGF- $\beta$ signalling. Nature 499, 306-311 (2013).

37. Zhang, Y., Luo, Q. \& Wang, N. et al. LRG1 suppresses the migration and invasion of hepatocellular carcinoma cells. Med. Oncol. 32, 146 (2015).

38. Byrum, R. S., Goulet, J. L. \& Griffiths, R. J. et al. Role of the 5-lipoxygenaseactivating protein (FLAP) in murine acute inflammatory responses. J. Exp. Med. 185, 1065-1075 (1997).

39. Kendall, R. T. \& Feghali-Bostwick, C. A. Fibroblasts in fibrosis: novel roles and mediators. Front. Pharmacol. 5, 123 (2014).

40. Man, J. \& Dytoc, M. T. Use of imiquimod cream $5 \%$ in the treatment of localized morphea. J. Cutan. Med. Surg. 8, 166-169 (2004).

41. Dytoc, M., Ting, P. T. \& Man, J. et al. First case series on the use of imiquimod for morphoea. Br. J. Dermatol. 153, 815-820 (2005).

42. Dytoc, M., Wat, H. \& Cheung-Lee, M. et al Evaluation of the efficacy and safety of topical imiquimod 5\% for plaque-type morphea: a multicenter, prospective, vehicle-controlled trial. J. Cutan. Med. Surg. 19, 132-139 (2015).

43. Campione, E., Paternò, E. J. \& Diluvio, L. et al. Localized morphea treated with imiquimod $5 \%$ and dermoscopic assessment of effectiveness. J. Dermatol. Treat. 20, 10-13 (2009).

44. Skare, T. L., Nisihara, R. M. \& Haider, O. et al. Liver autoantibodies in patients with scleroderma. Clin. Rheumatol. 30, 129-132 (2011).

45. Nay, J., Menias, C. O. \& Mellnick, V. M. et al. Gastrointestinal manifestations of systemic disease: a multimodality review. Abdom. Imaging 40, 1926-1943 (2015).

46. Serpier, H., Gillery, P. \& Salmon-Ehr, V. et al. Antagonistic effects of interferongamma and interleukin-4 on fibroblast cultures. J. Invest. Dermatol. 109, 158-162 (1997).

47. Gilbane, A. J., Denton, C. P. \& Holmes, A. M. Scleroderma pathogenesis: a pivotal role for fibroblasts as effector cells. Arthritis Res. Ther. 15, 215 (2013).

48. Clark, K. E., Lopez, H. \& Abdi, B. A. et al. Multiplex cytokine analysis of dermal interstitial blister fluid defines local disease mechanisms in systemic sclerosis. Arthritis Res. Ther. 17, 73 (2015).

49. Sfrent-Cornateanu, R., Mihai, C. \& Stoian, I. et al. Antioxidant defense capacity in scleroderma patients. Clin. Chem. Lab. Med. 46, 836-841 (2008).

50. Piera-Velazquez, S., Makul, A. \& Jiménez, S. A. Increased expression of NAPDH oxidase 4 in systemic sclerosis dermal fibroblasts: regulation by transforming growth factor $\beta$. Arthritis Rheumatol. 67, 2749-2758 (2015).

51. Piera-Velazquez, S. \& Jimenez, S. A. Role of cellular senescence and NOX4mediated oxidative stress in systemic sclerosis pathogenesis. Curr. Rheumatol. Rep. 17, 473 (2015). 
52. Spadoni, T., Svegliati Baroni, S. \& Amico, D. et al. A reactive oxygen speciesmediated loop maintains increased expression of NADPH oxidases 2 and 4 in skin fibroblasts from patients with systemic sclerosis. Arthritis Rheumatol. 67, 1611-1622 (2015).

53. Zhang, Y., McCormick, L. L. \& Desai, S. R. et al Murine sclerodermatous graftversus-host disease, a model for human scleroderma: cutaneous cytokines, chemokines, and immune cell activation. J. Immunol. 168, 3088-3098 (2002).

54. Wang, X., Hao, Y. \& Wang, X. et al. A PPARdelta-selective antagonist ameliorates $\mathrm{IMQ}$-induced psoriasis-like inflammation in mice. Int. Immunopharmacol. 40, 73-78 (2016).

55. Chamcheu, J. C., Chaves-Rodriquez, M. I. \& Adhami, V. M. et al. Upregulation of PI3K/AKT/mTOR, FABP5 and PPARbeta/delta in human psoriasis and imiquimod-induced murine psoriasiform dermatitis model. Acta Derm. Venereol. 96, 854-856 (2016).

56. Hack, K. Reilly, L. \& Palmer, C. et al. Skin-targeted inhibition of PPAR beta/delta by selective antagonists to treat PPAR beta/delta-mediated psoriasis-like skin disease in vivo. PLoS ONE 7, e37097 (2012).

57. Romanowska, M., Reilly, L. \& Palmer, C. N. et al. Activation of PPARbeta/delta causes a psoriasis-like skin disease in vivo. PLOS ONE 5, e9701 (2010)

58. Romanowska, M., al Yacoub, N. \& Seidel, H. et al. PPARdelta enhances keratinocyte proliferation in psoriasis and induces heparin-binding EGF-like growth factor. J. Invest. Dermatol. 128, 110-124 (2008).

59. Hawley, D. P., Pain, C. E. \& Baildam, E. M. et al. United Kingdom survey of current management of juvenile localized scleroderma. Rheumatology $\mathbf{5 3}$ 1849-1854 (2014).

60. van den Hoogen, F., Khanna, D. \& Fransen, J. et al. 2013 classification criteria for systemic sclerosis: an American College of Rheumatology/European League against Rheumatism collaborative initiative. Arthritis Rheum. 65, 2737-2747 (2013).

61. Takashima A. Establishment of fibroblast cultures. Curr. Protoc. Cell Biol. Chapter 2: Unit 2.1, pp 2.1.1-2.1.12, 2001.

62. Iglesias, J., Barg, S. \& Vallois, D. et al. PPARbeta/delta affects pancreatic beta cell mass and insulin secretion in mice. J. Clin. Invest. 122, 4105-4117 (2012).

63. Schuler, M., Ali, F. \& Chambon, C. et al. PGC1alpha expression is controlled in skeletal muscles by PPARbeta, whose ablation results in fiber-type switching, obesity, and type 2 diabetes. Cell Metab. 4, 407-414 (2006).

64. Trimboli, A. J., Cantemir-Stone, C. Z. \& Li, F. et al. Pten in stromal fibroblasts suppresses mammary epithelial tumours. Nature 461, 1084-1091 (2009).

65. Tan, C. K. Tan, E. H. \& Luo, B. et al. SMAD3 deficiency promotes inflammatory aortic aneurysms in angiotensin Il-infused mice via activation of iNOS. J. Am. Heart Assoc. 2, e000269 (2013).

66. Chong, H. C., Chan, J. S. K. \& Goh, C. Q. et al. Angiopoietin-like 4 stimulates STAT3-mediated iNOS expression and enhances angiogenesis to accelerate wound healing in diabetic mice. Mol. Ther. 22, 1593-1604 (2014).

67. Reddy, G. K. \& Enwemeka, C. S. A simplified method for the analysis of hydroxyproline in biological tissues. Clin. Biochem. 29, 225-229 (1996).

68. Tan, N. S. \& Wahli, W. Studying wound repair in the mouse. Curr. Protoc. Mouse Biol. 3, 171-185 (2013).

69. Yamamoto, T., Takagawa, S. \& Katayama, I. et al. Animal model of sclerotic skin. I: local injections of bleomycin induce sclerotic skin mimicking scleroderma. J. Invest. Dermatol. 112, 456-462 (1999).

70. Tan, N. S., Michalik, L. \& Di-Poi, N. et al. Essential role of Smad3 in the inhibition of inflammation-induced PPARbeta/delta expression. EMBO J. 23, 4211-4221 (2004). 